\title{
The Essence of the Elementary Charge and the Derivation and Calculation of the Electron Inertial Mass
}

\author{
Shlomo Barak ${ }^{1}$ \\ ${ }^{1}$ Taga Innovations 16 Beit Hillel St. Tel Aviv 67017, Israel \\ Correspondence: Shlomo Barak, Taga Innovations 16 Beit Hillel St. Tel Aviv 67017, Israel. E-mail: \\ shlomo@tagapro.com
}

Received: September 6, 2019

Accepted: September 27, $2019 \quad$ Online Published: September 30, 2019

doi:10.5539/apr.v11n5p26

URL: http://dx.doi.org/10.5539/apr.v11n5p26

\begin{abstract}
We consider Electric Charge (Barak, 2019) as merely a space deformation. Positive charge is a contracted zone of space and negative charge - a dilated zone. The field of the electric charge is a smooth continuation from this zone to infinity, of a diminishing contraction or dilation of space. This consideration enables us to derive theoretically, with no phenomenology, the Maxwell theory of electrostatics. Together with the Lorentz Transformation we derive (a known derivation procedure) Maxwell's theory in its entirety.

Our geometry of deformed zones of space (Barak, 2019), rather than Riemannian geometry of bent manifolds, enables us to attribute positive curvature to a contracted zone of space, i.e., to a positive charge, and negative curvature to a negative charge. This enables us to apply General Relativity (GR) in our derivations, and show that the positive elementary charge can be considered a kind of black hole, whereas the negative elementary charge - a white hole.

Based on the above we construct a model of the Electron (and other elementary particles) and derive and calculate its attributes, including inertial mass and spin. Neither the Standard Model nor String Theory has provided such results.

The equations for these masses contain only the constants $\mathrm{G}, \mathrm{c}, \hbar$ and $\alpha$ (the fine structure constant). Our calculated results comply with CODATA 2014.
\end{abstract}

Keywords: Electric Charge, Curved Space, Black Holes, Electron Mass, Quark Mass

\section{Introduction}

1.1 Our Result for the Inertial Mass of the Electron

$\mathrm{M}_{\mathrm{e}}=\frac{\mathrm{s}^{2} \sqrt{2}}{\pi(1+\pi \alpha)} \sqrt{\mathrm{G}^{-1} \alpha \hbar \mathrm{c}^{-3}}, \mathrm{~s}=1,[\mathrm{~s}]=\mathrm{LT}^{-1}$

$\mathrm{M}_{\mathrm{e}}($ calculated $)=0.910,3610^{-27} \mathrm{~g}$

$\mathrm{M}_{\mathrm{e}}($ measured $)=0.910,938,356(11) 10^{-27} \mathrm{~g}$

A dimensionality check: $\left[\mathrm{G}^{-1}\right]=\mathrm{ML}^{-3} \mathrm{~T}^{2},[\alpha]=1,[\hbar]=\mathrm{ML}^{2} \mathrm{~T}^{-1},\left[\mathrm{c}^{-3}\right]=\mathrm{L}^{-3} \mathrm{~T}^{3}$. Thus:

$\mathrm{M}=\left[\mathrm{s}^{2} \sqrt{\mathrm{G}^{-1} \alpha \hbar \mathrm{c}^{-3}}\right]=\mathrm{L}^{2} \mathrm{~T}^{-2}\left(\mathrm{ML}^{-3} \mathrm{~T}^{2} \cdot \mathrm{ML}^{2} \mathrm{~T}^{-1} \cdot \mathrm{L}^{-3} \mathrm{~T}^{3}\right)^{1 / 2}=\mathrm{L}^{2} \mathrm{~T}^{-2}\left(\mathrm{M}^{2} \mathrm{~L}^{-4} \mathrm{~T}^{4}\right)^{-1 / 2}=\mathrm{L}^{2} \mathrm{~T}^{-2} \mathrm{ML}^{-2} \mathrm{~T}^{2}=\mathrm{M}$

\subsection{The Bivalent Elementary Charges as Kind of Black and White Holes}

Susskind (2004) wrote:

One of the deepest lessons we have learned over the past decade is that there is no fundamental difference between elementary particles and black holes. As repeatedly emphasized by Gerard 't Hooft (1990), black holes are the natural extension of the elementary particle spectrum. This is especially clear in string theory where black holes are simply highly-excited string states. Does that mean that we should count every particle as a black hole?

Relating just to the elementary charge, our answer to this question is affirmative.

This idea was first expressed by Salam and Strathdee (1977), Holzhey and Wilczek (1992), and also Sen (1995). 
The intuition of these distinguished physicists is our inspiration.

Our attribution of curvature to an elementary charge, and the fact that charge is quantized, led us to pursue the possibility that positive and negative elementary charges are hitherto unrecognized kind of electrical black and white holes, respectively. This led us to the derivation and calculation, using cgs units, of the radii and masses presented in Section 1.6.

\subsection{Our Paper: The Electric Charge and Its Field as Deformed Space}

In the Barak (2019) paper, we show that charge is not alien to space but simply a drastically contracted or dilated zone of it. The electric field is a continuation from this zone to infinity, of a diminishing contraction or dilation. In this regard the field itself is also charge. We consider positive electric charge to be a contracted (deformed) zone of space and negative electric charge to be a dilated (deformed) zone of space. With this concept alone we derive theoretically, with no phenomenology, the Maxwell theory of electrostatics. Together with the Lorentz Transformation we derive Maxwell's theory in its entirety Barak (2017).

Note that, since the field is also a distribution of charge, the equations of our electromagnetism are non-linear and resemble QED. The approximation for a field of low strength yields linear equations, which are the Maxwell equations.

\subsection{Our Paper: On bent Manifolds and Deformed Spaces}

The geometry of deformed zones of space is our Barak (2019) extended Riemannian geometry, which originally is the geometry of bent manifolds. Hence, we can attribute positive curvature to a contracted zone of space, i.e., to a positive charge, and negative curvature to a negative charge.

We thus conclude that General Relativity (GR), which is formally a theory of bent manifolds of a continuous space, is actually a theory of a deformed elastic three-dimensional space lattice and four-dimensional space-time lattice. This realistic consideration enables us to apply GR in our derivations.

\subsection{The Current Paradigm in Physics}

The current paradigm is facing many obstacles. Many principles remain unproven, attributes of elementary particles cannot be derived and calculated, and mysteries are un-resolved. This situation results from the lack of a deeper theoretical layer.

\subsection{The Spacetime GeometroDynamic Model (GDM) of Reality}

Our GeometroDynamic Model (GDM) of Reality, see Appendix A, is this missing underlying layer. This layer, the GDM, provides answers as to what is charge, what is an elementary particle, and relates to additional fundamental subjects. Note that, at large, the GDM does not contradict the paradigm; it simply serves as a realistic and tangible substratum. Since this paper has a specific purpose, we do not elaborate on the GDM, but simply point out, whenever necessary, its relation to the present work. This work, however, exposes the reader to the ideas and reasoning of the GDM.

\subsection{Our Results for the Radii and Masses of Elementary Particles}

The radius $\mathbf{r}_{\mathbf{p}}$ of the Proton Charge (not the proton radius), as we show, is:

$r_{p}($ calculated $)=0.877410^{-13} \mathrm{~cm}$, which is well within the experimental error range Pohl (2010) $r_{p}($ measured $)=$ $0.8768(69) 10^{-13} \mathrm{~cm}$.

This is also the anti-proton charge radius that can be and should be measured, see Section 9.

Our result $r_{e}$ for the electron's inner circulation radius, based on, $r_{p}$, the proton charge radius, which is also the elementary charge radius $r_{Q}$, is:

$\mathrm{r}_{\mathrm{e}}=1.4098 \cdot 10^{-13} \mathrm{~cm}$

This $r_{e}$ is also the positron's inner circulation radius, see Section 9 .

Based on this $r_{\mathrm{e}}$ we derive and calculate the Mass $\mathbf{M}_{\mathrm{e}}$ of the Electron/Positron:

$\mathrm{M}_{\mathrm{e}}($ calculated $)=0.9103610^{-27} \mathrm{~g}$.

A deviation of only $0.063 \%$ from the measured CODATA value:

$\mathrm{M}_{\mathrm{e}}($ measured $)=0.910938356(11) 10^{-27} \mathrm{~g}$.

We also derive and calculate the Mass of the Muon/Anti-Muon.

$180.1510^{-6} \mathrm{erg}=112.5 \mathrm{MeV}$ or: 
$\mathrm{M}_{\mu}($ calculated $)=112.5 \mathrm{MeV} / \mathrm{c}^{2}$

According to CODATA 2014:

$\mathrm{U}_{\mu}($ experimental $)=105.6583745(24) \mathrm{MeV}$

$\mathrm{M}_{\mu}($ experimental $)=105.6583745(24) \mathrm{MeV} / \mathrm{c}^{2}$

Comparing the above results shows a deviation of $\sim 6 \%$ from the experimental CODATA value.

Based on $M_{e}$, and our Model of Quarks we derive and calculate, Barak (2016), the masses $\mathbf{M}_{\mathbf{d}}$ and $\mathbf{M}_{\widetilde{u}}$ of the d and $\widetilde{\mathrm{u}}$ Quarks:

$\mathrm{M}_{\mathrm{d}}=4.5 \mathrm{MeV} / \mathrm{c}^{2}$ recent experimental value $\mathrm{M}_{\mathrm{d}}=4.8+/-0.5 \mathrm{MeV} / \mathrm{c}^{2}$

$\mathrm{M}_{\widetilde{\mathrm{u}}}=2.25 \mathrm{MeV} / \mathrm{c}^{2}$ recent experimental value $\mathrm{M}_{\widetilde{\mathrm{u}}}=2.3+/-0.8 \mathrm{MeV} / \mathrm{c}^{2}$

See Fritzsch and Gell-Mann (2015) for the experimental measured masses.

So far, no other theory has provided such results.

\section{The Schwarzschild Black Hole and the Quantized Elementary Charge}

The Schwarzschild metric is the solution to the Einstein field equations of General Relativity (GR) outside a spherical mass. In spherical coordinates (t, r, $\theta, \varphi$ ), the line element for the Schwarzschild metric, Cheng (2015), is:

$$
\mathrm{ds}^{2}=\left(1-\frac{\mathrm{r}_{\mathrm{s}}}{\mathrm{r}}\right) \mathrm{c}^{2} \mathrm{dt}^{2}-\left(1-\frac{\mathrm{r}_{\mathrm{s}}}{\mathrm{r}}\right)^{-1} \mathrm{dr}^{2}-\mathrm{r}^{2} \mathrm{~d} \Omega^{2}
$$

where $\mathrm{c}$ is the speed of light, $\mathrm{t}$ is the time coordinate (measured by a stationary clock at infinity), $\mathrm{r}$ is the radial coordinate and $\mathrm{d} \Omega^{2}$ is a 2-sphere defined by: $\mathrm{d} \Omega^{2}=\mathrm{d} \theta^{2}+\sin ^{2} \theta \mathrm{d} \theta^{2}$.

The Schwarzschild, gravitational black hole radius, $r_{s}$, see the metric (1), in cgs units, is:

$$
\mathrm{r}_{\mathrm{s}}=\frac{2 \mathrm{GM}}{\mathrm{c}^{2}}
$$

or since $\mathrm{M}=\mathrm{U} / \mathrm{c}^{2}$ :

$$
\mathrm{r}_{\mathrm{s}}=\frac{2 \mathrm{GU}}{\mathrm{c}^{4}}
$$

Note that equation (2) is also the result of equating the potential energy GMM'/rs of a test mass $\mathrm{M}^{\prime}$, in the field of $\mathrm{M}$, to its ultimate kinetic energy of escape from the black hole $1 / 2 \mathrm{M}^{\prime} \mathrm{c}^{2}$.

We now show that (1) and (2) are also relevant to the bivalent elementary charges. The self-energy $U$ of a charge $\mathrm{Q}$, accumulated in a sphere with radius $r$ is $\mathrm{U}=\mathrm{Q}^{2} / 2$ r. This $\mathrm{U}$, Barak (2019) is the strain energy of a continuous space of both the charge and its field. In Appendix B (Please read it before you proceed) we set a virtual border, of radius $r$, to artificially divide equally the energy between the space zone of the charge and that of its field. This is our definition of $r$. Hence:

$\mathrm{r}=\mathrm{Q}^{2} / 4 \mathrm{U}$

However, for calculating the total energy $U$, of the charge and its field, and the mass $M$ of the electron we use again the relations: $\mathrm{U}=\mathrm{Q}^{2} / 2 \mathrm{r}$ and $\mathrm{M}=\mathrm{U} / \mathrm{c}^{2}$.

Inserting the above expression $r=Q^{2} / 4 U$ in (3) and notating $r=r_{s}$ gives the $r_{s}$ for a black hole:

$$
\mathrm{r}_{\mathrm{S}}=\frac{\mathrm{GQ}^{2}}{2 \mathrm{r}_{\mathrm{S}} \mathrm{c}^{4}}
$$

To explain the quantization of charge we suggest considering the elementary charge to be, not only a contraction or dilation of space, Barak (2019), but a black or white (respectively) hole. Thus, the radius of the elementary charge is related to the Schwarzschild radius. This consideration, as we show, yields the results mentioned in the Introduction.

By definition, an elementary charge curves space since it is the dilation or contraction of space, Barak (2019). This curving, as we show, is orders of magnitude larger than the gravitational curving by the energy/momentum of the particle, which is related to its spin, as we show elsewhere. 
The equality of the absolute values of the bivalent charges is related to their creating photon in the pair production process, Section 5 and Barak (2016).

Since (4) becomes $r_{s}^{2}=\frac{\mathrm{GQ}^{2}}{2 \mathrm{c}^{4}}$, its square root gives:

$r_{s}= \pm \sqrt{\frac{G^{2}}{2 c^{4}}}= \pm \frac{Q \sqrt{G}}{c^{2} \sqrt{2}}$ This $r_{s}$ is obtained for the self-energy of the elementary charge $Q$.

We re-notate it as $\mathrm{r}_{\mathrm{SQ}}$ and the above equation becomes:

$$
\mathrm{r}_{\mathrm{SQ}}= \pm \sqrt{\frac{\mathrm{GQ}^{2}}{2 \mathrm{c}^{4}}}= \pm \frac{\mathrm{Q} \sqrt{\mathrm{G}}}{\mathrm{c}^{2} \sqrt{2}}
$$

In (5) $r_{S Q}$ is positive for a positive charge and negative for a negative charge. A negative $\mathrm{r}_{\mathrm{SQ}}$ means a negative radius of curvature. In this case we have a "white hole" instead of a black hole or a "repulsion", as Kallosh and Linde (1995) using String Theory name it. This white hole reflects incoming particles like photons. The Curving by the Elementary Charge and its Schwarzschild Radius

The "gravitational" curving $K_{M}$ by a mass (energy), see Bergmann (1992) and Steane (2012), is given for $r \geq r_{S}$ by:

$$
\mathrm{K}_{\mathrm{M}}=1 / \mathrm{R}_{\mathrm{c}}{ }^{2}=\mathrm{r}_{\mathrm{S}} / \mathrm{r}^{3}
$$

$\mathrm{R}_{\mathrm{c}}$ is the Gaussian radius of curvature.

Using (2), the force $F_{M}$ that two equal masses $M$ apply on each other is:

$$
\mathrm{F}_{\mathrm{M}}=\frac{\mathrm{GM}^{2}}{\mathrm{r}^{2}}=\frac{\mathrm{c}^{4}}{4 \mathrm{G}} \frac{\mathrm{r}_{\mathrm{S}}^{2}}{\mathrm{r}^{2}}
$$

Thus, according to (6) and (7), force is related to space curvature.

In Sections 7 and 10 we show that the elementary charge mass is purely an electromagnetic mass. Here, at an earlier stage of our paper, we just assume that this the case.

Hence, we substitute in (7) the $r_{S Q}$ of (5), instead of $r_{s}$ of (2). This step is legitimate since we relate only to the "gravitational" curving due to mass/energy of the particle not to its charge; thus, the curvature's expression remains the same. This substitution gives:

$$
\mathrm{F}_{\mathrm{M}}=\frac{\mathrm{GM}^{2}}{\mathrm{r}^{2}}=\frac{\mathrm{c}^{4}}{4 \mathrm{G}} \frac{\mathrm{r}_{\mathrm{SQ}}^{2}}{\mathrm{r}^{2}}
$$

And (6) can be rewritten as:

$$
\mathrm{K}_{\mathrm{SQ}}(\text { energy })=1 / \mathrm{R}_{\mathrm{c}}{ }^{2}=\mathrm{r}_{\mathrm{SQ}} / \mathrm{r}^{3}
$$

We mow suggest expressing the electrical charge curving $\mathrm{K}_{\mathrm{Q}}$ by the net elementary charge (not the curving by its self-energy) in the same way as $\mathrm{K}_{\mathrm{M}}$ is expressed in (6). This is possible since charge is merely the curvature (deformation) Barak (2019) of space:

$$
\mathrm{K}_{\mathrm{Q}}(\text { charge })=1 / \mathrm{R}_{\mathrm{cc}}{ }^{2}=\mathrm{r}_{\mathrm{Q}} / \mathrm{r}^{3}
$$

where $r_{Q}$ to be the Schwarzschild radius of the net elementary charge, which is not the radius $r_{S Q}$ due to its self-energy. And $\mathrm{R}_{\mathrm{cc}}$ is the Gaussian radius of curvature of the net elementary charge. We assume that:

$$
\mathrm{r}_{\mathrm{Q}}=\mathrm{k} \mathrm{r}_{\mathrm{SQ}}
$$

Thus the force $\mathrm{F}_{\mathrm{Q}}$ that two equal charges $\mathrm{Q}$ apply on each other is, in similarity to (8):

$$
\mathrm{F}_{\mathrm{Q}}=\frac{\mathrm{Q}^{2}}{\mathrm{r}^{2}}=\mathrm{k}^{2} \frac{\mathrm{c}^{4}}{\mathrm{G}} \frac{\mathrm{r}_{\mathrm{SQ}}^{2}}{\mathrm{r}^{2}}
$$

The ratio of the forces $F_{Q}$ and $F_{M}$ becomes:

$$
\mathrm{F}_{\mathrm{Q}} / \mathrm{F}_{\mathrm{M}}=\frac{\mathrm{Q}^{2}}{\mathrm{GM}^{2}}=4 \mathrm{k}^{2}
$$

This ratio for the elementary charge $\mathrm{Q}_{\mathrm{e}}$ and the electron mass $\mathrm{M}_{\mathrm{e}}$ is: 


$$
\mathrm{F}_{\mathrm{Q}} / \mathrm{F}_{\mathrm{M}}=\frac{\mathrm{Qe}^{2}}{\mathrm{GMe}^{2}}=4.16210^{42}=\text { the numerical value of } 5.094 \mathrm{c}^{4}
$$

The numerical value of $\mathrm{c}^{4}$ can be written as $\mathrm{c}^{4} / \mathrm{s}^{4}$, where $\mathrm{s}=1$ and $[\mathrm{s}]=\mathrm{LT}^{-1}$. Thus, according to (13) and (14):

$$
\mathrm{k}^{2}=5.094 / 4 \mathrm{c}^{4} / \mathrm{s}^{4}=1.273 \mathrm{c}^{4} / \mathrm{s}^{4} \sim \mathrm{c}^{4} / \mathrm{s}^{4} \text { and } \mathrm{k}=1.128 \mathrm{c}^{2} / \mathrm{s}^{2} \sim \mathrm{c}^{2} / \mathrm{s}^{2}
$$

This result means that $r_{Q}$ (due to the intrinsic curvature of charge) is $\sim c^{2} / s^{2}$ larger than $r_{S Q}$ (due to the electromagnetic self-energy of the charge).

Regarding these Schwarzschild radii, we raise a conjecture. It leans on our assumption, equation (11), which is $\mathrm{r}_{\mathrm{Q}}=\mathrm{k} \mathrm{r}_{\mathrm{SQ}}$, and the experimental result (15) for the value of $\mathrm{k}$.

\section{Conjecture}

$\mathbf{r}_{\mathrm{Q}}$ (due to the intrinsic curvature of charge) $=\mathbf{r}_{\mathrm{sQ}}$ (due to the self-energy of charge) $\mathbf{c}^{2} / \mathbf{s}^{2}$

This conjecture for the relevant curvatures (9) and (10) is:

$$
K_{\mathbf{Q}}=\mathbf{c}^{2} / \mathbf{s}^{2} \mathbf{K} \mathbf{Q}
$$

By using (16); replacing $\mathrm{c}$ with $\mathrm{s}, \mathrm{s}=1,[\mathrm{~s}]=\mathrm{LT}^{-1}$, in the denominator of (5) our conjecture gives: The Elementary Charge's Radius

$$
\mathrm{r}_{\mathrm{Q}}=\frac{\sqrt{2} \mathrm{Q} \sqrt{\mathrm{G}}}{2 \mathrm{~s}^{2}}
$$

This relation (18) enables us to derive and calculate radii and masses of the elementary particles.

The proton charge radius (19), as we explain in Section 4, is also expressed by (18):

$$
r_{p}=\frac{\sqrt{2} Q \sqrt{G}}{2 s^{2}}
$$

\section{On the Proton's Charge Radius $\mathbf{r}_{\mathbf{p}}$}

\subsection{The Essence of the Proton's Charge Radius}

The radius of the proton's elementary charge was measured, but not that of the electron.

We relate to the proton because it enables us to compere our theoretical result (18) to measured values. And because this radius is needed in the understanding of the Pair Production, Section 5, and the structure of the electron Section 6.

In Barak (2016) we present a model of the quarks that construct the proton and derive and calculate their masses. We show that a quark is a "twisted" electron and the anti-quark - a "twisted" positron. This is why we consider the Proton's Charge Radius to be that of the elementary charge.

\subsection{The Calculated Versus the Experimental Results}

By substituting the value for $\mathrm{Q}$ in (18), as it appears in $\alpha=\mathrm{Q}^{2} / \hbar \mathrm{c}$, we get:

$$
r_{p}=\left(\sqrt{2} / 2 s^{2}\right) \sqrt{G \alpha \hbar c}
$$

Inserting the CODATA 2014 values $\mathrm{G}=6.67408(31) \cdot 10^{-8} \mathrm{~cm}^{3} \mathrm{gr}^{-1} \mathrm{sec}^{-2}$ and

$\mathrm{Q}=4.80320425(10) 10^{-10}$ esu in (19) gives:

$$
r_{p}(\text { calculated })=0.877410^{-13} \mathrm{~cm}
$$

This is the radius of the elementary charge and also that of the proton charge.

This result is well within the experimental error range, Pohl et al. (2010):

$$
\mathrm{r}_{\mathrm{p}} \text { (measured) }=0.8768(69) 10^{-13} \mathrm{~cm}
$$

This and the results presented in the next sections, confirm our conjecture.

The result (21) is also the anti-proton radius, see Section 9.

Our result (21) is somewhat larger than (22), the measured electronic hydrogen proton charge radius. And (22) is larger than that for the muonic hydrogen $0.84087(39) 10^{-13} \mathrm{~cm}$ Antognini, et al. (2013).

This discrepancy, between the measured results, is termed the "Proton radius puzzle" [17].

Note the possibility that it is the QED vacuum polarization that affects the measurements. Our result is for the 
bare proton whereas vacuum polarization in electronic hydrogen and muonic hydrogen affects the measurements to give smaller results. Note that the muon vacuum polarization is larger since the muon is about 200 times closer to the proton than the electron.

\section{On Pair Production}

Pair production is the creation of an elementary particle and its antiparticle, by the interaction of an energetic photon with matter. The electron and positron pair is an example.

In the GDM, and also in Loudon (2000), a photon is considered a transverse wavepacket whereas an elementary charge is considered a longitudinal circulating wavepacket (of contracted or dilated space) Barak (2019) A similar concept, suggested by Sakharov (1982) is titled: The Knot-Like Topological Structure of Elementary Charges. Pair production seems to be a mode conversion of the transverse wavepacket (the photon) into two longitudinal wavepackets rather than an extraction of an electron from Dirac's sea. This conversion takes place during the circulation of an, energetic enough, photon around a proton. This circulation is possible, since the proton is an electric black hole, which deforms (curves) space drastically. The photon spin 1 is divided equally between the particles (conservation of angular momentum) to attribute spin $1 / 2$ for each particle of the pair. Thus, the particles have to circulate on an additional larger circle than their inner circle, as is explained in Section 7.2.

\section{The Electron's Inner Radius of Circulation $r_{e}$}

\subsection{General}

In the GDM we relate to "rest" as the case in which a wavepacket moves in a closed loop. Hence, we consider the geometrical center, of the circulating wavepacket, as its space location at rest. Thus, from a distance, the electron (positron) is observed as if at rest, and from close, as a volumetric dilation (contraction), which is its negative (positive) charge that moves on a closed loop. This loop can be a simple circle or a compound loop of several circles.

The electron (positron) at "rest" is a circulating longitudinal dilatational (contractional) wavepacket with a tangential velocity of propagation $c_{\mathrm{L}}$ in its inner circulation with radius $\mathrm{r}_{\mathrm{e}}$, and an additional external circulation, with a tangential transversal velocity of propagation $c$, and radius $R_{e}$, Section 7.2. In this section we elaborate just on the inner circulation.

A photon, circulating an electric black hole like the proton charge, with energy $U$ above a certain threshold, can be converted into a pair. The photon, by this circulation, gains, Allen et al. (1999), orbital angular momentum $\mathrm{L}_{\mathrm{ph}}$, which is divided between the electron and positron, in the Pair Production:

$\mathrm{L}_{\text {photon }}=\mathrm{U} / \mathrm{c} \mathrm{r}_{\text {proton }}=\mathrm{L}_{\text {electron }}+\mathrm{L}_{\text {positron }}$ and since:

$\mathrm{U}=\mathrm{U}_{\mathrm{e}}+\mathrm{U}_{\mathrm{p}}=2 \mathrm{U}_{\mathrm{e}}$ the orbital angular momentum gained by the electron is:

$$
\mathrm{L}_{\mathrm{eo}}=\mathrm{U}_{\mathrm{e}} / \mathrm{c} \mathrm{r}_{\mathrm{p}}
$$

The numerical value for $\mathrm{L}_{\mathrm{eo}}$ is $2.393610^{-30} \mathrm{erg}$ sec. Note that $\mathrm{L}_{\mathrm{eo}}$ is perpendicular to the $\mathrm{k}$ vector of the photon and, hence, to its spin. Thus, the intrinsic angular momentum of the electron $1 / 2 \hbar$ plus the additional perpendicular $\mathrm{L}_{e}$ should create a larger magnetic dipole moment. This, unknown, classical result gives a correction to the magnetic dipole moment which is similar to the one-loop correction of the QED calculation. Dividing our result for $\mathrm{L}_{\mathrm{eo}}$ by the Dirac factor 2 , and by $\hbar$, gives our correction which is: 0.001135 , whereas that of Schwinger (1948), the one-loop correction, is: $\alpha / 2 \pi=0.0011614$.

$\mathrm{L}_{\mathrm{eo}}$ for the electron, as a longitudinal wavepacket with wave velocity $\mathrm{c}_{\mathrm{L}}$, is:

$$
\mathrm{L}_{\mathrm{eo}}=\mathrm{U}_{\mathrm{e}} / \mathrm{c}_{\mathrm{L}} \mathrm{r}_{\mathrm{e}}
$$

Note that $r_{e}$ (the inner circulation radius of the electron) is not $r_{Q}$ (the radius of its charge), which is $r_{p}$.

Equating (23) and (24) gives:

$$
r_{e}=c_{L} / c r_{p}
$$

The value of $r_{e}$ gives, as (26) shows, the self-energy of the electron $U_{e}$ and hence its equivalent mass $M_{e}$ :

$$
\mathrm{U}_{\mathrm{e}}=\mathrm{Q}_{\mathrm{e}}{ }^{2} /\left(2 \mathrm{r}_{\mathrm{e}}\right)
$$

Using $(25,26)$ to derive and calculate $U_{e}$ and $M_{e}$ requires the derivation and calculation of $c_{L}$, which appears in (25). This is done in the next section.

\subsection{Derivation and Calculation of the Longitudinal Wavepacket Velocity $c_{L}$ and Feynman's Self Interactions}

QED uses the concept of virtual photons and Feynman Diagrams, to perform accurate calculations. The idea is 
that particles exchange virtual photons and can also self-interact. This section shows how a classical approach to self-interaction can yield a result for $\mathrm{c}_{\mathrm{L}}$.

The buildup of a field around a suddenly created charge propagates, from the charge onwards, at velocity c. The sudden destruction of the charge causes the field around it to vanish at the same speed. Imagine, now, a circulating charge that moves with velocity $\mathrm{c}_{\mathrm{L}}$, where $\mathrm{c}_{\mathrm{L}}>\mathrm{c}$.

In this case, if to move along half a circle takes less time than for the field to vanish along the diameter, the charge will be affected by its own field created when it passed the opposite point on the diameter, as if the charge, still exist on the other side of the diameter. We refer to this virtual charge as the image of the charge. Self-interaction is thus the interaction of a charge with its image, as we phrase it. Imagine the electron charge circulating with velocity $c_{L}$ around a point. Self-interaction occurs if the time to circulate a half circle, $\pi \mathrm{r}_{\mathrm{e}} / \mathrm{c}_{\mathrm{L}}$, is shorter than, or equal to, the time for its propagating or retreating field to cross the diameter $2 r_{e} / c$. Thus, selfinteraction takes place if at least:

$\pi r_{e} / c_{L}=2 r_{e} / c$. The requirement for self-interaction is thus:

$$
\mathrm{c}_{\mathrm{L}} / \mathrm{c} \geq \pi / 2
$$

The minimal value of this ratio is:

$$
\mathrm{c}_{\mathrm{L}} / \mathrm{c}=\pi / 2
$$

By constructing a more accurate model of the electron (Section 7.2), we arrive at (38), which is more accurate than the ratio (28).

\subsection{The Stability of the Self-Circulation and the Anomalous Gyroscopic Moment}

The self-circulation is kept stable by the attractive Lorentz force $\mathbf{F}=\mathrm{Q} / \mathrm{c} \mathbf{v x} \mathbf{B}$, towards the center of the circulation. The magnetic field $\mathbf{B}$, which the charge senses, is created by the circulation of its image. This force balances the repulsive force $\mathrm{F}=\mathrm{Q} \cdot \mathrm{Q} /\left(2 \mathrm{r}_{\mathrm{e}}\right)^{2}$ between the charge and its image. The magnetic field created by the circulating charge, [7], is $\mathbf{B}=1 / \mathrm{c} \mathbf{v x E}$. Hence: $\mathbf{F}=(\mathrm{Q} / \mathrm{c}) \mathbf{v x} \mathbf{B}=(\mathrm{Q} / \mathrm{c}) \mathbf{v x}(1 / \mathrm{c}) \mathbf{v x} \mathbf{E}$ but $\mathbf{v}=\mathbf{c}$ and this equation becomes $\mathbf{F}=\mathrm{Q} \mathbf{E}$ or $\mathrm{F}=\mathrm{Q} \cdot \mathrm{Q} /\left(2 \mathrm{r}_{\mathrm{e}}\right)^{2}$ This centripetal force balances the repulsive force above. Note that this calculation is not dependent on whether we use $\mathrm{c}$ or $\mathrm{c}_{\mathrm{L}}$.

The anomalous gyroscopic moment is considered a quantum mechanical phenomenon that cannot be explained by classical physics.

Note, however, that the circulation of the self-interacting charge with its own "image" is a current of "two" charges that double $\mathbf{E}$ and hence double $\mathbf{B}=1 / \mathrm{c}$ vxE. These "two" charges are circulating along the larger circle of radius $R_{e}$ related to the spin, see Section 7.2. We wonder if this is the cause for the factor 2 in the anomalous gyroscopic moment, as Dirac showed. The additional Schwinger (1948) correction factor $\alpha / 2 \pi$ is discussed in Section 7.2.

\subsection{The Approximated Electron's Inner Circulation Radius $r_{e}$}

Substituting the ratio (28) in (25) gives:

$$
r_{e}=c_{L} / c r_{p}=\pi / 2 r_{p}
$$

Using (19) we get:

$$
r_{\mathrm{e}}=\frac{\pi \sqrt{2}}{4 \mathrm{~s}^{2}} \sqrt{\mathrm{G}} \mathrm{Q}
$$

Replacing $\mathrm{Q}$ in (30) by its value derived from the Fine Structure Constant $\alpha=\mathrm{Q}^{2} / \hbar \mathrm{c}$, gives:

$$
\mathrm{r}_{\mathrm{e}}=\frac{\pi \sqrt{2}}{4 \mathrm{~s}^{2}} \sqrt{\mathrm{G} \alpha \hbar \mathrm{c}}
$$

The numerical value for $\mathrm{r}_{\mathrm{e}}$, based on CODATA 2014, is:

$$
\mathrm{r}_{\mathrm{e}}=1.37810^{-13} \mathrm{~cm}
$$

A more accurate result appears in Section 7.2.

Note that the inner radius of circulation of the electron is larger than the radius of its elementary charge: (32) versus (21). 


\section{The Electron Mass}

\subsection{An Approximation Taking $c_{L} / c=\pi / 2$}

Using (26) and (30) we obtain the equation for the mass of the electron:

$$
\mathrm{M}_{\mathrm{e}}=\frac{\mathrm{s}^{2} \sqrt{2}}{\pi} \frac{\mathrm{Q}}{\mathrm{c}^{2} \sqrt{\mathrm{G}}}
$$

Replacing $\mathrm{Q}$ in (33), by its value derived from $\alpha=\mathrm{Q}^{2} / \hbar \mathrm{c}$, (33) becomes:

$$
\mathrm{M}_{\mathrm{e}}=\frac{\mathrm{s}^{2} \sqrt{2}}{\pi} \sqrt{\mathrm{G}^{-1} \alpha \hbar \mathrm{c}^{-3}}
$$

The numerical values for $r_{e}, U_{e}$ and $M_{e}$ are (for more accurate results see Section 7.2):

$$
r_{\mathrm{e}}=1.37810^{-13} \mathrm{~cm}
$$

Substituting this result in $\mathrm{U}_{\mathrm{e}}=\mathrm{Q}_{\mathrm{e}}{ }^{2} /\left(2 \mathrm{r}_{\mathrm{e}}\right)$, which is (26), gives:

$$
\mathrm{U}_{\mathrm{e}}=0.83710^{-6} \mathrm{erg}
$$

Dividing by $\mathrm{c}^{2}$ (and not $\mathrm{c}_{\mathrm{L}}^{2}$ ) gives:

$$
\mathrm{M}_{\mathrm{e}}=0.9311910^{-27} \mathrm{gr}
$$

This is a $\sim 2 \%$ deviation from the experimental value.

\subsection{An Accurate Derivation and Calculation and the External Circulation Radius $R_{e}$}

To retain exactly the $1 / 2 \hbar$ angular spin momentum, contributed by the photon in the pair production process, the elementary charge has to participate in an additional circulation. This circulation with velocity $\mathrm{c}$ and radius $\mathrm{R}_{\mathrm{e}}$, gives: $1 / 2 \hbar=\mathrm{U}_{\mathrm{e}} / \mathrm{c} \mathrm{R}_{\mathrm{e}}$, and should be incorporated in our model to complete it. The complete model of circulations is described as follows:

The charge circulates with an "inner" radius $r_{e}$ and velocity $c_{L}$ around the circumference of the spin circle with an "external" radius $\mathrm{R}_{\mathrm{e}}$, and describes an epicycloid. The velocity of propagation of this charge along the circumference of this circle of radius $R_{e}$ is, however, $c$ and not $c_{L}$, since its rotation while traveling around the circumference slows down the actual propagation velocity. By reaching this compound lower velocity $\mathrm{c}$, the electron gains stability, and is unable to radiate, Section 6.3 and Appendix D.

We therefore modify $c_{L} / c$ in (28) to become $c_{L} / c=\pi\left(R_{e}+\pi r_{e}\right) / 2 R_{e}$ or:

$$
\mathrm{c}_{\mathrm{L}} / \mathrm{c}=\pi / 2\left(1+\pi \mathrm{r}_{\mathrm{e}} / \mathrm{R}_{\mathrm{e}}\right)
$$

Using (24) and $1 / 2 \hbar=\mathrm{U}_{\mathrm{e}} / \mathrm{c} \mathrm{R}_{\mathrm{e}}$ we get:

$$
r_{e} / R_{e}=\alpha,
$$

where $\alpha$ is the Fine Structure Constant, hence:

$$
\begin{gathered}
\mathrm{c}_{\mathrm{L}} / \mathrm{c}=\pi / 2(1+\pi \alpha) \\
\mathrm{c}_{\mathrm{L}} / \mathrm{c}=\mathrm{r}_{\mathrm{e}} / \mathrm{r}_{\mathrm{p}}=1.6068
\end{gathered}
$$

According to (25), (38) and (19) the corrected relation for $\mathrm{r}_{\mathrm{e}}$ is:

$$
\mathrm{r}_{\mathrm{e}}=\pi / 2(1+\pi \alpha) \sqrt{2} / 2 \mathrm{~s}^{2} \sqrt{\mathrm{G}} \mathrm{Q}
$$

or:

$$
\mathrm{r}_{\mathrm{e}}=\frac{\pi \sqrt{2}}{4 \mathrm{~s}^{2}}(1+\pi \alpha) \sqrt{\mathrm{G} \alpha \hbar \mathrm{c}}
$$

Inserting the CODATA 2014 values in (41) gives:

$$
r_{e}(\text { calculated })=1.4098510^{-13} \mathrm{~cm}
$$

This is also the GDM radius of the Positron, not yet measured experimentally, see Section 9.

Substituting this improved relation (41) for $\mathrm{r}_{\mathrm{e}}$ in equation (26), $\mathrm{U}_{\mathrm{e}}=\mathrm{Q}_{\mathrm{e}}{ }^{2} /\left(2 \mathrm{r}_{\mathrm{e}}\right)$, gives:

$$
\mathrm{U}_{\mathrm{e}}=0.8181910^{-6} \mathrm{erg}
$$

Dividing the two sides of (26) by $\mathrm{c}^{2}$ and using (40), gives: 


$$
\mathrm{M}_{\mathrm{e}}=\frac{\mathrm{s}^{2} \sqrt{2}}{\pi(1+\pi \alpha)} \frac{\mathrm{Q}_{\mathrm{e}}}{\mathrm{c}^{2} \sqrt{\mathrm{G}}}
$$

Using $\alpha=Q^{2} / \hbar c$ to replace $Q$ in (44) gives:

$$
\mathrm{M}_{\mathrm{e}}=\frac{\mathrm{s}^{2} \sqrt{2}}{\pi(1+\pi \alpha)} \sqrt{\mathrm{G}^{-1} \alpha \hbar \mathrm{c}^{-3}}
$$

CODATA 2014 values for $\alpha, \hbar, G$, and c inserted in (45) give the numerical value for $\mathrm{M}_{\mathrm{e}}$ :

$$
\mathrm{M}_{\mathrm{e}}(\text { calculated })=0.9103610^{-27} \mathrm{~g}
$$

a deviation of $\sim 0.063 \%$ from the experimental CODATA value:

$\mathrm{M}_{\mathrm{e}}($ measured $)=0.910938356(11) 10^{-27} \mathrm{gr}$.

This result confirms our conjecture.

According to (39): $\mathrm{r}_{\mathrm{e}} / \mathrm{r}_{\mathrm{p}}=\mathrm{r}_{\mathrm{e}} / \mathrm{r}_{\mathrm{Q}}=1.6077$.

7.3 The Relation of $M_{e}$ to $M_{\text {Planck }}$

$\mathrm{M}_{\text {Planck }}=\sqrt{\mathrm{G}^{-1} \hbar \mathrm{c}}=2.176470(51) 10^{-5} \mathrm{gr}$

Hence:

$$
M_{e}=\frac{s^{2} \sqrt{2}}{\pi(1+\pi \alpha)} \sqrt{G^{-1} \alpha \hbar c^{-3}}=\frac{s^{2} \sqrt{2 \alpha}}{\pi(1+\pi \alpha) c^{2}} M_{\text {Planck }}
$$

\section{The Derivation and Calculations of the Muon and Tau Masses}

\subsection{The Muon}

A photon that enters the pair production process, by circulating the proton charge, might be converted into the heavy muon and anti-muon pair. This happens if the energy $U_{\text {photon }}$ is high enough to retain the angular momentum of the particle/anti-particle $\mathrm{L}=1 / 2 \hbar$, by circulation with radius $\mathrm{r}_{\mathrm{e}}$ and velocity $\mathrm{c}_{\mathrm{L}}$, around the proton charge alone and with no need for an additional larger circulation with radius $\mathrm{R}_{\mathrm{e}}$. Hence:

$1 / 2 \hbar=\left(\mathrm{U}_{\mu} / \mathrm{c}_{\mathrm{L}}\right) \mathrm{r}_{\mathrm{e}}$ or:

$$
\mathrm{U}_{\mu}=1 / 2 \hbar \mathrm{c}_{\mathrm{L}} / \mathrm{r}_{\mathrm{e}}
$$

Using (39) for $c_{L}$ and (42) for $r_{e}$ we get:

$\mathrm{U}_{\mu}($ calculated $)=0.51 .054571800(13) 10^{-27} 1.60682 .9979245810^{10} / 1.4098510^{-13}=$

$180.1510^{-6} \mathrm{erg}=112.5 \mathrm{MeV}$ or:

$$
\mathrm{M}_{\mu}=112.5 \mathrm{MeV} / \mathrm{c}^{2}
$$

According to CODATA 2014:

$\mathrm{U}_{\mu}($ experimental $)=105.6583745(24) \mathrm{MeV}$

$$
\mathrm{M}_{\mu}(\text { experimental })=105.6583745(24) \mathrm{MeV} / \mathrm{c}^{2}
$$

Comparing (48) to (49), shows a deviation of $\sim 6 \%$ from the experimental CODATA value.

\subsection{The Tau}

According to the CODATA 2014:

$$
\mathrm{U}_{\tau} / \mathrm{U}_{\mu}=1776.84 / 105.66=16.82
$$

$\mathrm{L}=\mathrm{P} \cdot \mathrm{R}=\left(\mathrm{U}_{\tau} / \mathrm{c}^{\prime}\right) \mathrm{r}$ and if $\mathrm{L}$ is retained then $\mathrm{r} / \mathrm{c}^{\prime}$ should be $(1 / 16.82) \mathrm{r}_{\mu} / \mathrm{c}_{\mathrm{L}}$ or:

$\mathrm{r} / \mathrm{c}^{\prime}=(1 / 16.82) 1.37810^{-13} / 1.5348310^{10}=1.77910^{-25} \mathrm{sec}$. This result occurs if $\mathrm{r}<\mathrm{r}_{\mu}$, which means that the elementary charge rotates around an inside point. But for rotation inside a black or white hole $c^{\prime}<c_{L}$. Thus, $r$ might be even smaller than $r_{\mu} / 16.82$.

Note that the elementary charge of both the tau and the muon moves at the longitudinal velocity and there is no circulation that brings the overall average velocity down to that of the transverse velocity, c. In this case, the charge moves faster than the photons it radiates, which explains the short life-time of these particles. In the case of the electron, the compound circulation of the charge brings down the average velocity to the light velocity, c. Hence, photons cannot be radiated (or radiated but re-absorbed). This explains the stability of the electron, 


\section{Section 6.3.}

\section{On the Radii of the Anti-Proton and Positron}

In Section 2.2 of Barak (2019) we define electric charge density as $q=\frac{1}{4 \pi} \frac{\rho-\rho_{0}}{\rho}$, where $\rho$ is the space density. Hence the electric charge in a given zone of space $\tau$ is: $Q=\int_{\tau} q d \tau$, where $[q]=1$ and $[Q]=L^{3}$. The factor $1 / 4 \pi$ is introduced to ensure resemblance to the Gaussian system and for no other reason.

For the spherical symmetric case, where $\mathrm{d} \tau=4 \pi \mathrm{r}^{2} \mathrm{dr}$, this gives the following result for $\mathrm{Q}$ :

$\mathrm{Q}=\int_{0}^{\mathrm{r}} \mathrm{q} 4 \pi \mathrm{r}^{2} \mathrm{dr}=1 / 3 \mathrm{r}^{3}\left(1-\rho_{0} / \rho\right)=\mathrm{V}-\mathrm{V}^{\prime}$, where $\mathrm{V}^{\prime}=\mathrm{V} \rho_{0} / \rho$. Thus $\rho>\rho_{0}$ gives $\mathrm{V}>\mathrm{V}^{\prime}$ and $\mathrm{Q}>0$, whereas $\rho<$ $\rho_{0}$ gives $\mathrm{V}<\mathrm{V}^{\prime}$ and $\mathrm{Q}<0$.

In a given spherical zone of space with radius $r$, as we outsider observers measure, positive charge means more space cells in this zone than in an un-deformed space, whereas negative charge means less space cells in this zone than in an un-deformed space.

Note that the equality $\left|Q_{+}\right|=\left|Q_{-}\right|$, of the absolute values of the bivalent elementary charges, means $\left|Q_{+}\right|=\left|Q_{-}\right|=\mid$ $\mathrm{V}-\mathrm{V}^{\prime} \mid$. It also means that $\left(1-\rho_{0} / \rho_{+}\right)=-\left(1-\rho_{0} / \rho_{-}\right)$and hence

$$
2 / \rho_{0}=1 / \rho_{+}+1 / \rho
$$

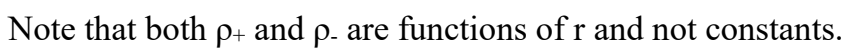

Since the mass of elementary particles equals that of their anti-particles and the energy is purely electromagnetic, we conclude that their radii are also equal.

This conclusion means that the radius of the Proton's charge is also that for the Anti-Proton's charge and that the radius of the Electron's charge is also that for the Positron's.

The radius of the Anti-Proton charge can be measured, using the same spectroscopic methods as are used to measure the Proton's charge radius, see Pohl (2010).

\section{The Electron in Motion and Lorentz Transformation}

\section{1 "Rest" and Motion in the GDM}

Every disturbance in space must move at the velocity of its elastic waves, $\mathrm{c}_{\mathrm{L}}$ or $\mathrm{c}_{\mathrm{T}}$.

A transverse disturbance in space moves at the speed of light $\mathrm{c}=\mathrm{c}_{\mathrm{T}}$, whereas a longitudinal disturbance moves at a higher speed $\mathrm{c}_{\mathrm{L}}$.

Note that a longitudinal wavepacket is necessarily a moving dilation, contraction or an oscillation between the two.

As a consequence, there is no state of rest. "Rest" is defined, therefore, as a situation in which a disturbance, although moving at velocity $\mathrm{c}_{\mathrm{L}}$ or $\mathrm{c}_{\mathrm{T}}$, is on a closed track. This orbital movement, Dirac's Zitterbewegung, is the spin of elementary particles.

On constant translational velocity v, and accelerated motion, relative to space, see Section 11.3.

\subsection{Spin and Angular Momentum}

Figure (1) is a simple description of the electron (positron) at "Rest" and in Motion. The gray sphere (circle) represents the zone of drastically dilated (contracted) space, which is the electric charge. In this discussion we omit, for simplicity, the inner circulation of the charge, with radius $r_{e}$. We also do not show in Figure (1) that the circulation plane is not perpendicular to the velocity vector $\mathbf{v}$. The projection of the angular momentum $\mathbf{L}$, of this circulation, on $\mathbf{v}$ is $L_{z}=1 / 2 \hbar$ whereas $L=\hbar \sqrt{j(j+1)}=\hbar \sqrt{1 / 2(1 / 2+1)}=0.866 \hbar$, hence the inclination of the circulation plane.

"Rest" is described on the left of Figure (1). The zone of dilated space (charge) of our electron revolves, in the xy plane, around the origin with a radius $\mathrm{R}_{0}$ ( in the previous sections we notated this radius as $\mathrm{R}_{\mathrm{e}}$ ). In this case: $\mathrm{c}$ 
$=\omega R_{0}$. From now on, when we relate to the electron we also relate to the positron, unless otherwise is mentioned.

\subsection{Motion}

For simplicity we ignore in the following sections the inner circulation of the electric charge.

Figure (1), on the right, describes a translational motion. Motion is the situation in which the circle of revolution of the wavepacket becomes a spiral. On this spiral the length of a revolution is retained, since angular momentum is retained (explained below). Hence the spiral radius $\mathrm{R}$ is smaller than $\mathrm{R}_{0}$. This is in analogy to a stretched spring. According to Figure (1):

$$
\mathrm{R}=1 / \gamma \mathrm{R}_{0}
$$

Note that $\mathrm{R}_{0}$ is our $\mathrm{R}_{\mathrm{e}}$ in the previous sections.

The resultant electron motion, however, is always at the wave velocity c. Thus, a translational motion at constant velocity, $v$, does not involve any exertion of force (a proof of Newton's first law). It is also obvious that necessarily $\mathrm{v}<\mathrm{c}$.

From this alone we derive similar results to that of the Theory of Special Relativity (SR), but without SR.

The length of a revolution is retained despite the reduction in $\mathrm{R}$ since angular momentum is retained. And the angular momentum is retained, see Section 11, since the energy is increasing.

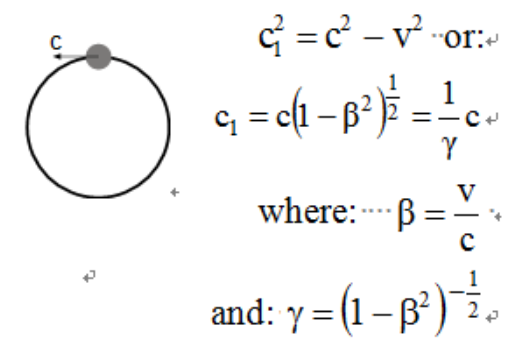

"Rest"

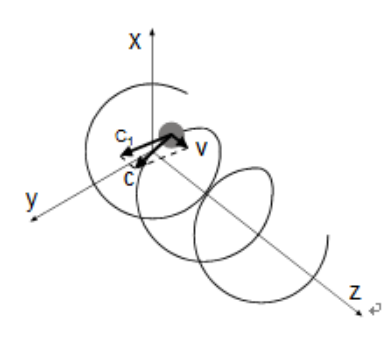

Motion

Figure 1. Rest and Motion

\subsection{Lorentz Transformation (LT)}

Our model of the moving electron yields the LT. In a way, it is similar to the contracted particle in motion, used by Lorentz (1904) to derive his LT. Note that both models attribute the LT to the contraction of particles due to their motion. This contraction is also the reason for the slowing down of the rates of clocks. But all this is out of the scope of this paper.

Newton's first law, and the SR conclusion that no particle or signal can move at a speed that exceeds the light/wave velocity c, are thus a natural result of our model. The helical motion, which is an electric current formally in the direction - z, is related to the magnetic field $\mathbf{B}$ and the vector potential $\mathbf{A}$. The Circular Track is stable since the centrifugal force is balanced by an equal but opposite centripetal force. The Lorentz force, created by the magnetic field of the circulating charge acting on itself, is the required centripetal force, Sec 7.3

\section{The Electron Inertia}

At rest, the energy of the electron is $U_{0}$, linear momentum is $P_{o}=U_{0} / c$ and the angular momentum is $L_{0}=P_{0} R_{0}$. In motion, energy, linear momentum and angular momentum are $\mathrm{U}, \mathrm{P}=\mathrm{U} / \mathrm{c}$ and $\mathrm{L}=\mathrm{PR}$ respectively.

The conservation of angular momentum $\mathrm{L}=\mathrm{L}_{0}$ (which is $1 / 2 \hbar$ for the electron) implies that:

$\mathrm{PR}=\mathrm{P}_{\mathrm{o}} \mathrm{R}_{\mathrm{o}}$ hence $\mathrm{UR}=\mathrm{U}_{0} \mathrm{R}_{0}$ but:

$$
\mathrm{R}=1 / \gamma \mathrm{R}_{0}
$$

Thus:

$$
\mathrm{U}=\gamma \mathrm{U}_{0}
$$


Considering the energy as purely electromagnetic, where the elementary charge $\mathrm{Q}$ is a sphere of radius $\mathrm{r}_{0}$, the relations $\mathrm{U}_{0}=\mathrm{Q}^{2} / 2 \mathrm{r}_{0}, \mathrm{U}=\mathrm{Q}^{2} / 2 \mathrm{r}$ and (2) give:

$$
\mathrm{r}=1 / \gamma \mathrm{r}_{0}
$$

For $\mathrm{v}<<\mathrm{c}(53)$ is:

$$
r=\left(1-v^{2} / 2 c^{2}\right) r_{0}
$$

Acceleration is accompanied by an increase of energy $\mathrm{U}$, and a reduction in the radii $\mathrm{R}$ and $\mathrm{r}$. The applied force, needed to accelerate the particle, is doing work to curve space more strongly and thus to reduce $r$. This force is needed to increase stress and cause more strain in order to enlarge the curvature. Thus, we arrive at Newton's Second Law and understand Inertia.

Note that we have replaced Newton's axiomatic laws by our postulated model of an elementary particle. Some of the merits in this replacement, as we show here and elsewhere, are:

- It leads to the proof of the equivalence of gravitational mass and inertial mass. And, more than that, it shows that gravitational mass is inertial mass.

- It proves that mass is not a fundamental attribute of matter.

- It proves that inertia is an intrinsic attribute of matter, with no need for an additional field to induce it (Higgs Field).

Note that $\mathrm{L}=1 / 2 \hbar, \mathrm{R}=1 / 2 \quad \hbar \mathrm{c} / \mathrm{U}, \mathrm{U}_{0}=\mathrm{Q}^{2} / 2 \mathrm{r}_{0}$ and $\mathrm{r}=\mathrm{Q}^{2} / 2 \mathrm{U}$ give:

$$
\alpha=r / R=Q^{2} / \hbar c \text {, Fine Structure Constant. }
$$

This result reveals the meaning of $\alpha$.

Note also that at "rest" the angular momentum can point in any direction, whereas in motion it can point, in the direction of motion or opposite to it. This attribute is related to Space Quantization.

\section{Relativistic Mass}

Our "relativistic" relation (52) gives:

$$
\mathrm{U}=\gamma \mathrm{U}_{0}=\mathrm{U}_{0}\left(1-\frac{\mathrm{v}^{2}}{\mathrm{c}^{2}}\right)^{-\frac{1}{2}} \simeq \mathrm{U}_{0}+\frac{\frac{1}{2} \mathrm{U}_{0} \mathrm{v}^{2}}{\mathrm{c}^{2}} \text {, The second term: } \frac{\frac{1}{2} \mathrm{U}_{0} \mathrm{v}^{2}}{\mathrm{c}^{2}}=1 / 2\left(\mathrm{U}_{0} / \mathrm{c}^{2}\right) \mathrm{v}^{2} \text {, is identified as the }
$$

kinetic energy where:

$$
\mathrm{M}_{0}=\mathrm{U}_{0} / \mathrm{c}^{2}
$$

Thus, inertial mass becomes merely a practical term. We also get:

$$
\mathrm{M}=\gamma \mathrm{M}_{0}
$$

The literature distinguishes between the kinetic energy $1 / 2 M_{0} v^{2}$, which a particle possesses by virtue of its

motion, and its internal energy $\mathrm{M}_{0} \mathrm{c}^{2}$. But here, the kinetic energy is also an internal energy of deformation (the reduction in size of $\mathrm{r}$ and $\mathrm{R}$ ) that cannot be distinguished from the rest energy.

Note that our model for the moving electron, presented here, uses the simplified version of the much more detailed model of the electron at "rest" that appears in section 7.2.

More on our electron is presented in: Appendix C - The Moving Electron - Supposedly a "Point-Like" Particle. Appendix D - Disintegration and Radiation of and by the Electron.

\section{Conclusions}

Our idea that electric charge and its field are a contracted or dilated zone of space led us to attribute curvature to electric charge and enabled us to use General Relativity. This is the basis of our derivation and calculation of the radii and masses of the elementary particles. Our result for the mass of the electron is $\mathrm{M}_{\mathrm{e}}=0.91036 \cdot 10^{-27} \mathrm{gr}$; a deviation of only $0.06 \%$ from the experimental value. Neither the Standard Model nor String Theory has provided such results. All our results are based on the attribution of pure electromagnetic self-energy to the 
elementary particles. We show that inertia is also the result of pure electromagnetic self-energy and structure. We thus conclude that mass is not a fundamental attribute of matter but just a practicality.

\section{Acknowledgements}

We would like to thank Professor Y. Silberberg of the Weizmann Institute of Science, for his careful reading and helpful comments, and Mr. Roger M. Kaye for his linguistic contribution and technical assistance.

\section{Conflict of interests}

The authors declare that there is no conflict of interests regarding the publication of this paper.

\section{References}

Allen et al. (1999). The orbital angular momentum of light: E. Wolf. Progress in Optics, XXXIX, 291.

Antognini et al. (2013). Science, 339, 417.

Barak, S. (2016). The Photon and the Quantum Enigma. Retrieved from https://hal.archives-ouvertes.fr/hal-01423548

Barak, S. (2016). Where is Anti-Matter? Retrieved from https://hal.archives-ouvertes.fr/hal-01423547

Barak, S. (2017). Electromagnetism as the Geometrodynamic of Space. Retrieved from https://hal.archives-ouvertes.fr/hal-01498448

Barak, S. (2018). A Spacetime Geometrodynamic Model(Gdm) Of The Physical Reality. Retrieved from https://hal.archives-ouvertes.fr/hal-01935260

Barak, S. (2019). Electric Charge and its Field as Deformed Space. Applied Physics Research, 11(4).

Barak, S. (2019). On Curved Manifolds and Deformed Spaces. Journal of Mathematics Research, 11(4).

Bergmann, P. G. (1992). The Riddle of Gravitation (p. 195).

Bourilkov, D. (2001). Phys. Rev. D, 64, 071701R.

Cheng, T. P. (2005). Relativity, Gravitation and Cosmology. Oxford.

Feynman, R. (1965). Lectures on Physics, 2(8-6).

Fritzsch, H., \& Gell-Mann, M. (Eds.) (2015). 50 Years of Quarks, World Scientific.

Holzhey, C. F. E., \& Wilczek, F. (1992). Black Holes as Elementary Particles.

Kallosh, R., \& Linde, A. (1995). Exact supersymmetric massive and massless white holes. Phys. Rev., D52, 7137.

Loudon, R. (2000). The Quantum theory of light. Oxford University Press.

Pohl et al. (2010). The size of the proton. Nature, 466(7303), 213-6.

Sakharov, A. D. (1982). The knot-like topological structure of elementary charges, Collection of scientific works (Vol. 4). Moscow, Nauka.

Salam, A., \& Strathdee, J. (1977). Hadronic temperature and black solitons. Physics Letters B, 66(2), 143-146.

Schwinger, J. (1948). On Quantum-Electrodynamics and the Magnetic Moment of the Electron. Physical Review, 73(4), 416.

Sen, A. (1995). Extremal black holes and elementary string states. Modern Physics Letters A. World Scientific.

Steane, A. M. (2012). Relativity Made Relatively Easy (p. 276). Oxford.

Susskind, L. (2004). arxiv.org/abs/hep-th/0407266.

Thooft, G. (1990). Nuclear Physics, B335, 138-154.

\section{Appendix A}

\section{On the GDM}

The Spacetime GeometroDynamic Model, or in short, the GDM, Barak (2018), is our realistic and tangible model of Reality

\section{The GDM Idea}

The Elastic and Vibrating three-dimensional Space Lattice is all there is. 
Elementary Particles are Transverse or Longitudinal Wavepackets of the vibrating space.

\section{The Units of the GDM}

In the GDM all units are expressed by the unit of length $\mathrm{L}(\mathbf{c m})$ and the unit of time $\mathrm{T}(\mathbf{s e c})$ only. A conversion from the cgs system of units to the GDM system and vice versa is possible.

\section{The Constants of Nature According to the GDM}

$\mathrm{c}_{\mathrm{T}}=\mathrm{c}$ Velocity of transverse Space vibrations (EM waves) $\quad\left[\mathrm{c}_{\mathrm{T}}\right]=\mathrm{LT}^{-1}$

$\left[\mathrm{c}_{\mathrm{L}}\right.$ Velocity of longitudinal Space vibrations $\left.\left(\mathrm{c}_{\mathrm{L}}>\mathrm{c}_{\mathrm{T}}\right)\right] \quad\left[\mathrm{c}_{\mathrm{L}}\right]=\mathrm{LT}^{-1}$

$\hbar$ Planck Constant $\quad[\hbar]=\mathrm{L}^{5} \mathrm{~T}^{-1}$

G Gravitational Constant $\quad[\mathrm{G}]=\mathrm{T}^{-2}$

$\alpha$ Fine Structure Constant $\quad[\alpha]=1$

Note that in the GDM (see also [1]): $[\mathrm{v}]=\mathrm{LT}^{-1},[\mathrm{a}]=\mathrm{LT}^{-2},[\mathrm{H}]=[\mathrm{G}]=\mathrm{T}^{-2},[\mathrm{Q}]=[\mathrm{M}]=\mathrm{L}^{3},\left[\mathrm{E}_{\mathrm{E}}\right]=\left[\mathrm{E}_{\mathrm{G}}\right]=\mathrm{LT}^{-2}$, $\left[\varphi_{\mathrm{E}}\right]=\left[\varphi_{\mathrm{G}}\right]=\mathrm{L}^{2} \mathrm{~T}^{-2},[\mathrm{~F}]=\mathrm{L}^{4} \mathrm{~T}^{-2},[\mathrm{U}]=\mathrm{L}^{5} \mathrm{~T}^{-2}$.

Note that $c_{L} / c=\pi / 2(1+\pi \alpha)$, see (38), and we can exclude $c_{L}$ from the list.

\section{"Rest" and Motion in the GDM}

Every disturbance in space must move at the velocity of its elastic waves, $\mathrm{c}_{\mathrm{L}}$ or $\mathrm{c}_{\mathrm{T}}$.

A transverse disturbance in space moves at the speed of light $\mathrm{c}=\mathrm{c}_{\mathrm{T}}$, whereas a longitudinal disturbance moves at a higher speed $\mathrm{c}_{\mathrm{L}}$

Note that a longitudinal wavepacket is necessarily a moving dilation, contraction or an oscillation between the two. As a consequence, there is no state of rest. "Rest" is defined, therefore, as a situation in which a disturbance, although moving at velocity $\mathrm{c}_{\mathrm{L}}$ or $\mathrm{c}_{\mathrm{T}}$, is on a closed track. This orbital movement, Dirac's Zitterbewegung, is the spin of elementary particles.

A "translational" motion of this wavepacket at a constant velocity v, relative to space, is the motion on a spiral with a constant length of a revolution.

An accelerated motion is a motion on a spiral, with an ongoing contraction of its radius.

The GDM considers the mass of a particle to be just a practicality, since inertia depends only on the energy of the particle.

\section{Appendix B}

\section{Is the Electrostatic Energy in the Charge or in the Field?}

This long-standing open issue, addressed by Feynman (1965) and others, is: The energy needed to create a charge $\mathrm{Q}$ of radius $\mathrm{r}$, by bringing in from infinity infinitesimal amounts of charge, despite repulsion, is:

$\mathrm{U}=\mathrm{Q}^{2} / 2 \mathrm{r}$

The energy density of the electrostatic field $\mathrm{E}=\mathrm{Q} / \mathrm{r}^{2}$ is:

$\epsilon=1 / 4 \pi \mathrm{E}^{2}$

and the entire energy in the field is also: $\mathrm{U}=\int_{r}^{\infty} \epsilon \mathrm{d} \tau=\mathrm{Q}^{2} / 2 \mathrm{r}$

Thus, we ask where is the energy, in the field or in the charge?

Our model of the elementary electric charge solves this issue.

In our model there is no separation between the charge and its field; they are a continuous deformation of space Einstein's vision. The above calculated energy, in both cases, is the same total energy of the charge and its field.

Thus, $r$ can only be considered as an artificial "border" between the two. We define - freely invent (Einstein's expression) - this $r$ to be a virtual border for which on both of its sides resides the same half of the above $U$.

Hence, for $r$ calculation we take $r=Q^{2} / 4 U$.

Note that $\mathrm{U}$ is the space energy of strain, be it contraction or dilation. 


\section{Appendix C}

\section{Size of the Moving Electron}

Scattering experiments with energies of $10 \mathrm{TeV}$ (at the LEP storage ring), "sets" an "upper limit" of $\sim 210^{-18} \mathrm{~cm}$ for the electron size Bourilkov (2001). In these experiments, the higher the scattering energy, the shorter the distance between the colliding particles (by overcoming the repulsion). According to the GDM the imparted energy, in the experiment, reduces the radius of the electron, and there is almost no lower limit to its size, see Figure (1C).

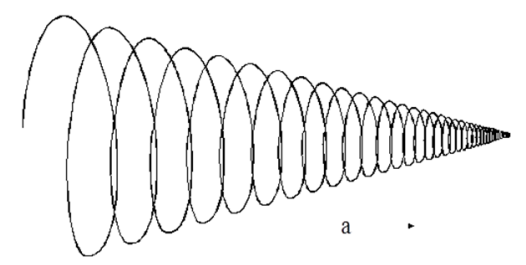

Figure (1C). The track of the Accelerated Particle

\section{Appendix D}

\section{No Disintegration Neither Radiation of and by the Electron Stability}

Considering the electron as a classical particle that can stay at rest, raises the question regarding the electrostatic repulsion that can tear it apart.

In the GDM, however, the electron is a wavepacket that always moves at the speed of light, whether on a closed loop ("rest") or an open spiral (motion). Therefore, to address the issue of stability, we should consider the Lorentz force between the "parts of an electron", each with charge $\delta \mathrm{Q}$, that happen to run in parallel and at the speed of light.

The Lorentz force: $\mathbf{F}=\delta \mathrm{Q}\left(\mathbf{E}+\frac{1}{\mathrm{c}} \mathbf{v} \times \mathbf{B}\right)$ is the force that "one part of the electron", with charge $\delta \mathrm{Q}$, applies on "another part", also with charge $\delta Q$, by creating the fields $\mathbf{E}$ and $\mathbf{B}$. Using the expression $\mathbf{B}=(1 / \mathrm{c})$ $\mathbf{v} \times \mathbf{E}$, that defines $\mathbf{B}$, and taking $\mathrm{v}=\mathrm{c}$, we get $\mathbf{F}=0$. This result explains stability.

\section{Radiation}

In both the "rest" and linear motion modes, the electron charge moves at a tangential speed $\mathrm{c}$ on a closed circle or a spiral. This might be expected to yield synchrotron radiation, and a loss of energy. But this radiation moves at the speed of light, which is also the speed of the "supposedly emitting charge" and hence can never leave it.

\section{Copyrights}

Copyright for this article is retained by the author(s), with first publication rights granted to the journal.

This is an open-access article distributed under the terms and conditions of the Creative Commons Attribution license (http://creativecommons.org/licenses/by/4.0/). 